\title{
Investigation of Macrobending Losses of Standard Single Mode Fiber with Small Bend Radii
}

\author{
Pengfei Wang \\ Technological University Dublin, pengfei.wang@tudublin.ie \\ Qiang Wu \\ Technological University Dublin, qiang.wu@tudublin.ie \\ Gerald Farrell \\ Technological University Dublin, gerald.farrell@tudublin.ie
}

See next page for additional authors

Follow this and additional works at: https://arrow.tudublin.ie/engscheceart

Part of the Engineering Commons

\section{Recommended Citation}

Wang, P. et al. (2007) "Investigation of macrobending losses of standard single mode fiber with small bend radii", Microwave and Optical Technology Letters, Vol. 49, Issue 9, September 2007, pp. 2133-213. doi;10.1002/mop.22671

This Article is brought to you for free and open access by the School of Electrical and Electronic Engineering at ARROW@TU Dublin. It has been accepted for inclusion in Articles by an authorized administrator of ARROW@TU Dublin. For more information, please contact arrow.admin@tudublin.ie, aisling.coyne@tudublin.ie, gerard.connolly@tudublin.ie.

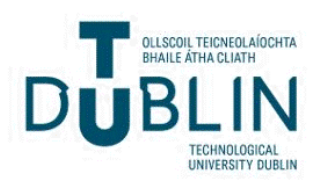




\section{Authors}

Pengfei Wang, Qiang Wu, Gerald Farrell, Ginu Rajan, Thomas Freir, and John Cassidy 


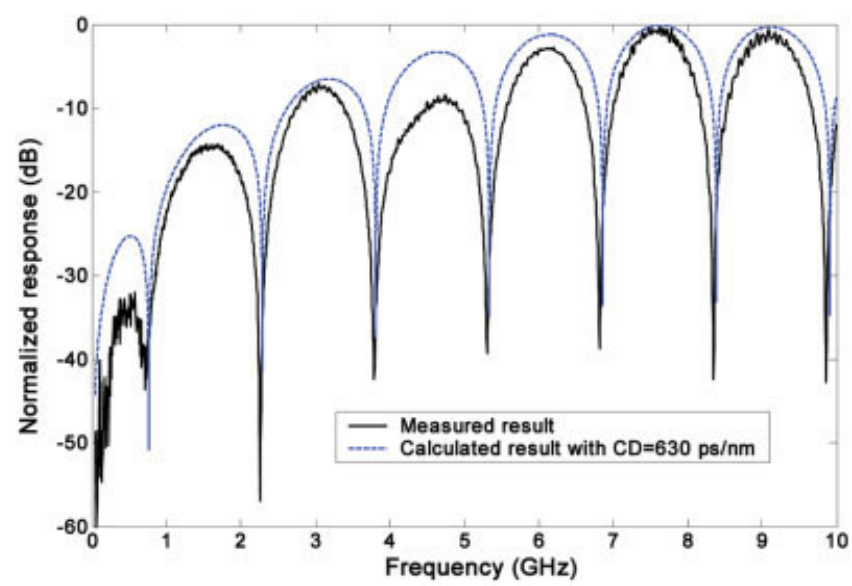

Figure 3 Measured filter response for narrow the bandwidth to $2 / 3$ of the original LCFBG. [Color figure can be viewed in the online issue, which is available at www.interscience.wiley.com]

When the chirped FBG bandwidth is narrowed to $1.02 \mathrm{~nm}$ with fixed centre wavelength at $1564.44 \mathrm{~nm}$, the $\mathrm{CD}$ values of the chirped FBG becomes $630 \mathrm{ps} / \mathrm{nm}$, which is 1.5 time of the original $\mathrm{CD}$ values of the LCFBG. Based on Eq. (6), it shows that the envelope response, $\sin \left(d_{\rho} \omega_{\mathrm{m}}^{2} \lambda^{2} / 4 \pi c\right)$, will increase. Figure 3 shows the calculated result and the measured filter response in dot line and solid line, respectively. Further, when the bandwidth is almost half of the original LCFBG, the CD value of the LCFBG is almost doubled. The envelope response of the filter is even stronger, as shown in Figure 4.

\section{CONCLUSIONS}

Bandpass photonic microwave filter has been demonstrated using a section of Hi-Bi fiber and a tunable LCFBG. The LCFBG serves as a tunable dispersion element, together with the phase modulator to eliminate the baseband resonance. The bandpass filter is free from the problem of optically coherent interference and phase noise. And the adjustment of the bandpass filter response by tuning the CD values of the LCFBG has also been achieved. Measured results agree with the theoretical analysis.

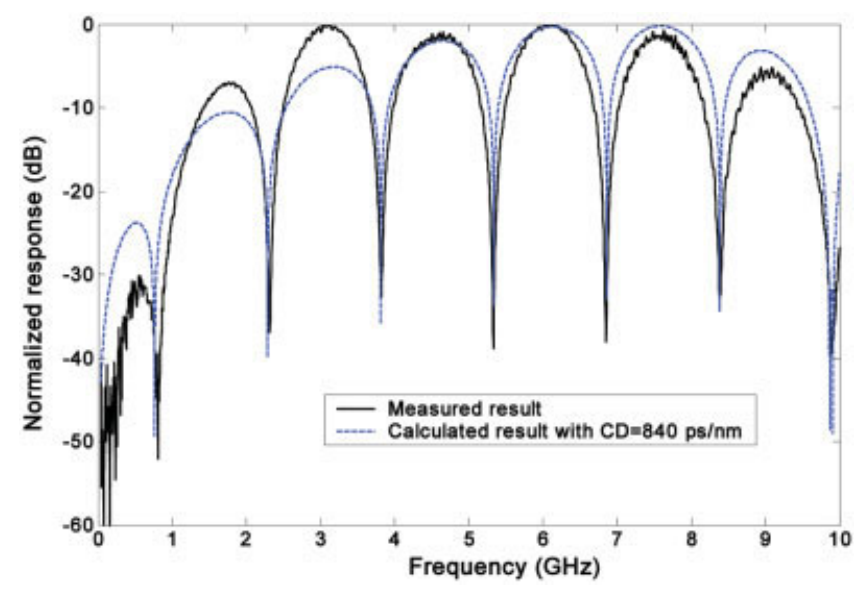

Figure 4 Measured filter response for narrow the bandwidth to half of the original LCFBG. [Color figure can be viewed in the online issue, which is available at www.interscience.wiley.com]

\section{REFERENCES}

1. J. Capmany, B. Ortega, D. Paster, and S. Sales, Discrete-time optical processing of microwave signlas, J Lightwave Technol 23 (2005), $702-723$.

2. R.A. Minasian, Photonic signal processing of microwave signals, Trans Microwave Theroy Tech 31 (2006), 832-846.

3. D. Paster and J.Capmany, Fibre optic tunable transversal filter using laser array and linearly chirped fibre grating, Electron Lett 34 (1998), $1684-1685$.

4. E.H.W. Chan, K.E. Alameh, and J.A.R. Minasian, Photonics bandpass filters with high skirt selectivity and stopband attenuation, J Lightwave Technol 20 (2002), 1962-1967.

5. W. Zhang, J.A.R. Williams, and I. Bennion, Optical fiber delay line filter free of limitation imposed by optical coherence, Electron Lett 35 (1999), 2133-2134.

6. J. Wang, and J.P. Yao, All-optical microwave bandpass filters implemented in a Radioover-fiber link, Photon Technol Lett 17 (2005), 1737-1739.

7. X. Dong, J. H. Ng, N. Q. Ngo, and P. Shum, CFBG-based tunable dispersion compensator with fixed central wavelength, In European Conference on Optical Communication, Paper We4, 2003, pp. 7-8.

8. A.R. Chraplyvy, R.W. Tkach, L.L. Buhl, and R.C. Alferness, Phase modulation to amplitude modulation conversion of CW laser light in optical fibers, Electron Lett 22 (1986), 409-410.

9. E. Turan, Fiber grating spectra, J Lightwave Technol 15 (1997), 12771294.

(C) 2007 Wiley Periodicals, Inc.

\section{INVESTIGATION OF MACROBENDING LOSSES OF STANDARD SINGLE MODE FIBER WITH SMALL BEND RADII}

\author{
Pengfei Wang,, ${ }^{1}$ Qian Wang, ${ }^{1}$ Gerald Farrell, ${ }^{1}$ Ginu Rajan, ${ }^{1}$ \\ Thomas Freir, ${ }^{1}$ and John Cassidy ${ }^{2}$ \\ ${ }^{1}$ Applied Optoelectronics Center, School of Electronics and \\ Communication Engineering, Dublin Institute of Technology, Kevin \\ Street, Dublin 8, Ireland \\ ${ }^{2}$ School of Chemical and Pharmaceutical Sciences, Dublin Institute \\ of Technology, Kevin Street, Dublin 8, Ireland
}

Received 9 February 2007

ABSTRACT: An investigation of macrobending loss characteristics of a standard single mode fiber (SMF28) for small bend radii is presented theoretically and experimentally, which includes the bend loss of the SMF28 with coating layers and the bare SMF28 after stripping the coating layers and chemical etching of partial cladding. The significant influence of reflection occurring at the interface between the cladding and coating layer or the cladding layer and air on the bend loss is investigated theoretically and experimentally. (c) 2007 Wiley Periodicals, Inc. Microwave Opt Technol Lett 49: 2133-2138, 2007; Published online in Wiley InterScience (www.interscience.wiley.com). DOI 10.1002/ mop.22671

Key words: fiber macrobending loss; single mode fiber; whispering gallery mode

\section{INTRODUCTION}

Optical fiber has been used in a range of optical sensing applications involving microbending or macrobending [1-6]. Examples include displacement sensing [1], pressure sensing [2, 4], wavelength referencing sensing [3], temperature sensing [6], and so on. Theoretical investigations about macrobending loss of fibers started in 1970s. The models developed by Marcuse [7, 8] treated the fiber as a core-infinite cladding structure. For the core-clad- 
ding-infinite coating structure, a number of theoretical modeling and corresponding experimental investigations of macrobending loss have been presented in Refs. [9-13], which considered the impact of the whispering-gallery mode (WGM) caused by the reflection of the radiated field at the interface between the cladding and coating layer. Previous published investigations of fiber bend losses have been focused on some special fibers (particularly fibers with small numerical apertures) rather than standard single mode fibers (such as SMF28) that are widely used in optical communications [9-12].

Recently, the macrobending loss properties of SMF28 (bend radius ranges from 8.5 to $12 \mathrm{~mm}$ ) were investigated theoretically and experimentally and optimized as an edge filter for wavelength measurements [14, 15]. However, a bending fiber with a smaller bend radius, e.g., substantially less than $10 \mathrm{~mm}$, is useful for sensing applications, particularly when the fiber bend is optimized as a small optical probe. Therefore, it is necessary to study the characteristics of macrobending loss with smaller bend radii. In practice, after stripping the polymer coating layers, the bare fiber is easily broken without any protection. To reduce the bending induced internal stress and allow for smaller bending fiber structures, the fiber cladding is etched partially by using HF acid, which will be presented in Section 4.

A thorough investigation of the fiber bend loss with small bend radii is presented theoretically and experimentally, which includes (1) theoretical modeling analysis for fiber bend loss; (2) macrobending loss of the SMF28 with coating layers; (3) macrobending loss for the bare SMF28 fiber after stripping the coating layers (core-cladding structure only) and partially etching the cladding layer; (4) macrobending loss of the bare etched SMF28 fiber after coating with an absorbing layer. The theoretical results agree with the measured bend losses for SMF28 with or without polymer coating layers. Through comparison between the first two cases and the third case, it is found that the WGM caused by reflection

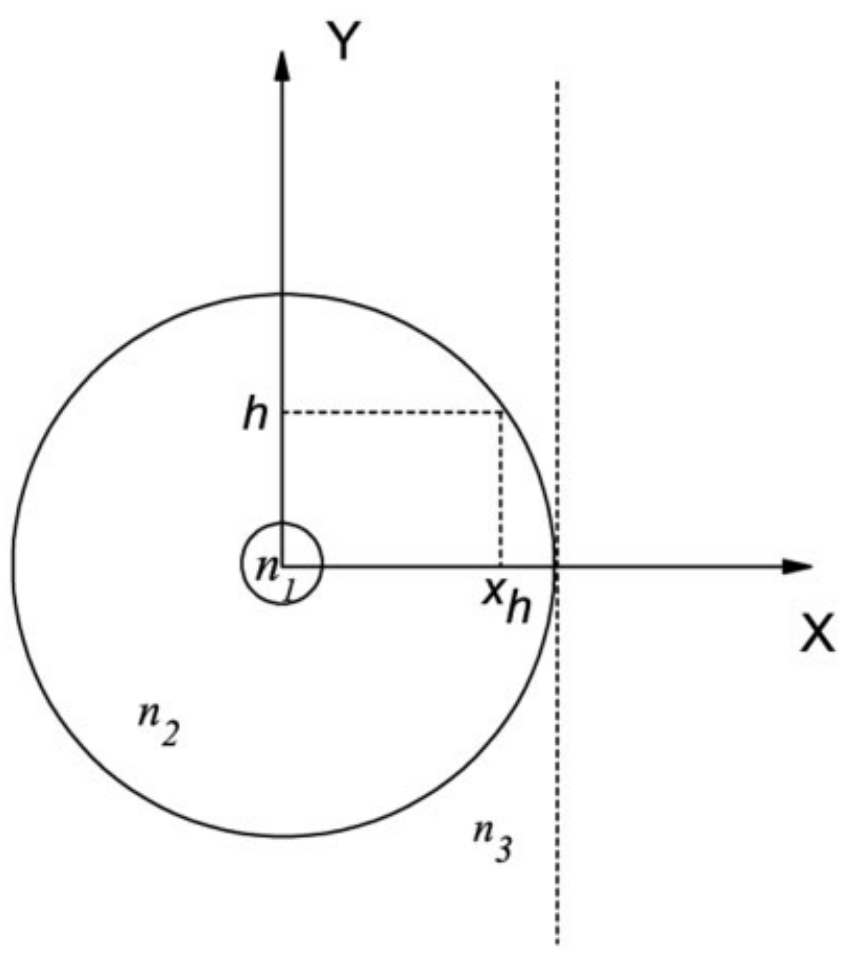

Figure 1 The cross section view of the bend fiber with core-claddinginfinite coating structure
TABLE 1 Parameters of the Standard Corning SMF28 Fiber; (the Refractive Index Values are Defined At a Wavelength of $1550 \mathrm{~nm})$

\begin{tabular}{lcc}
\hline SMF28 fiber & Refractive index & Radius $(\mu \mathrm{m})$ \\
\hline Core & $n_{1}=1.4504$ & $a=4.15$ \\
Cladding & $n_{2}=1.4447$ & $b=62.5$ \\
Inner coating & $n_{3}=1.4786$ & $c=80$ \\
Outer coating & $n_{4}=1.5294$ & $d=125$ \\
\hline
\end{tabular}

at the interface between the cladding and coating layer (Case 1) or between the cladding layer and air (Case 2) have a significant impact on the bend loss.

\section{THEORETICAL MODELING FOR FIBER BEND LOSS}

There are different approaches developed for the prediction of the macrobending loss of single mode fibers with coatings [10-12]. For example, a theoretical model based on weak perturbance of the guide field has been presented in Ref. [10-12]. Figure 1 illustrates the cross section of a bend fiber with a core-cladding-infinite coating layer structure. Based on the weak-guidance approximation theory, when the fiber is bent, the Fourier transform scalar field in the cladding and infinite coating regions in both $x$ and $y$-direction can be expressed as a Fourier series as follows [10]:

$\Psi(x, y)$

$$
= \begin{cases}\sum_{p=1}^{N}\left\{C_{\rho} B i\left[X_{2, \rho}(x)\right]+R_{\rho} A i\left[X_{2, \rho}(x)\right]\left\{\cos \beta_{\rho} y \quad a \leq x \leq x_{h}\right.\right. \\ \sum_{p=1}^{N} D_{P}\left\{B_{i}\left[X_{3, \rho}(x)\right]-i A i\left[X_{3, \rho}(x)\right]\right\} \cos \beta_{\rho} y & b \leq x<\infty\end{cases}
$$

Assuming that bending takes place in the $x$-plane, $\beta_{p}$ is the conjugate variable for the Fourier transform in the restricted narrow $y$-region, and $\beta_{p}$ could be expressed as: $\beta_{\rho}=(2 \rho$ $-1) \pi / 2 h$ ( $p$ is positive integer, $p=1,2, \ldots$ ), and $h$ is

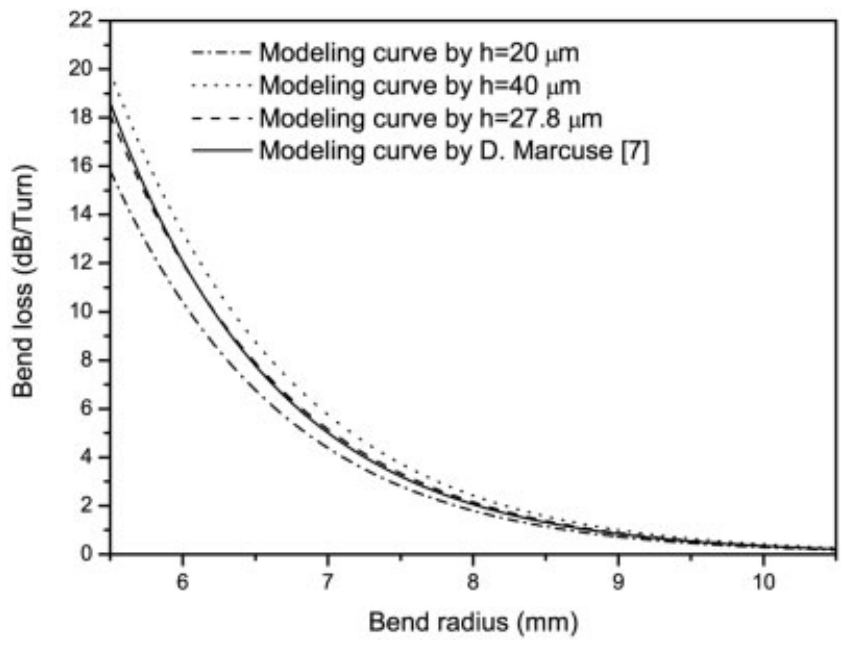

Figure 2 Theoretical modeling bend loss curves from Ref. [7] (solid line) and Ref. [10] (the dashed line is with $h=27.8 \mu \mathrm{m}$; the dash-dot line is with $h=20 \mu \mathrm{m}$; the dotted line is with $h=40 \mu \mathrm{m}$ ) for SMF28 fiber with different bend radii at the wavelength is $1500 \mathrm{~nm}$ 
defined in Figure 1. In our calculations, it is found that the calculated bend loss differs with the $h$ value. A simple and practical method to find out a suitable $h$-value can be (1) assume the refractive index of the coating to equal to that of the cladding, i.e., core-infinite cladding structure, (2) calculate the bend loss with the above method and the method developed by Marcuse in Ref. [7], separately under different bending radii, (3) determine a suitable $h$-value so that the two results match.
For the Corning standard SMF28 fiber considered in this article, the refractive indices (for wavelength $1550 \mathrm{~nm}$ ) and radii of the core, cladding, and coating layers are shown in Table 1.

Figure 2 shows the calculated fiber bend loss results from Ref. [7] and [10] for standard SMF28 fiber. For illustration, values of $h$ equal to $20,27.8$, and $40 \mu \mathrm{m}$ for the $y$-direction are shown. From Figure 2, one can see that the calculated result with $h=27.8 \mu \mathrm{m}$ (dashed line) from Ref. [10] is in agreement

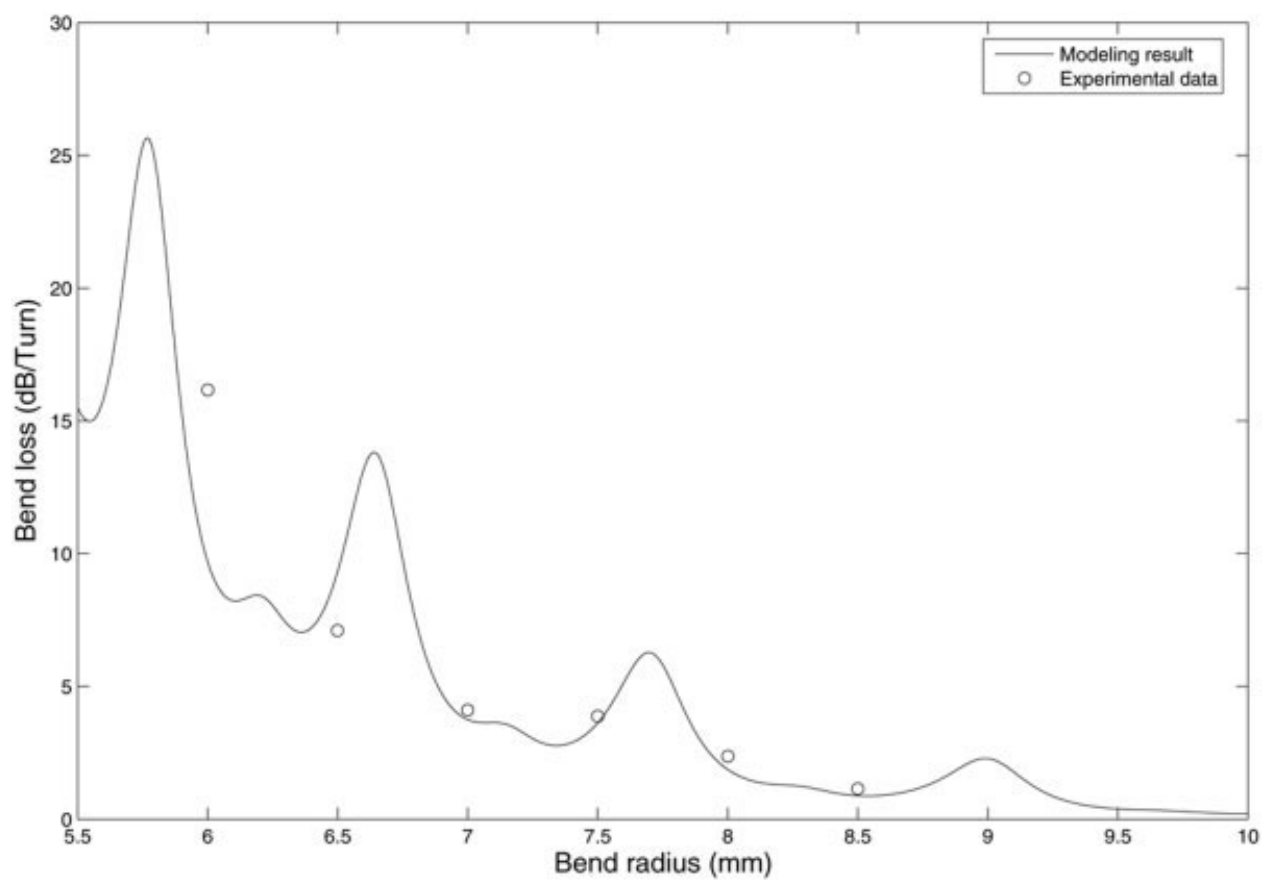

(a)

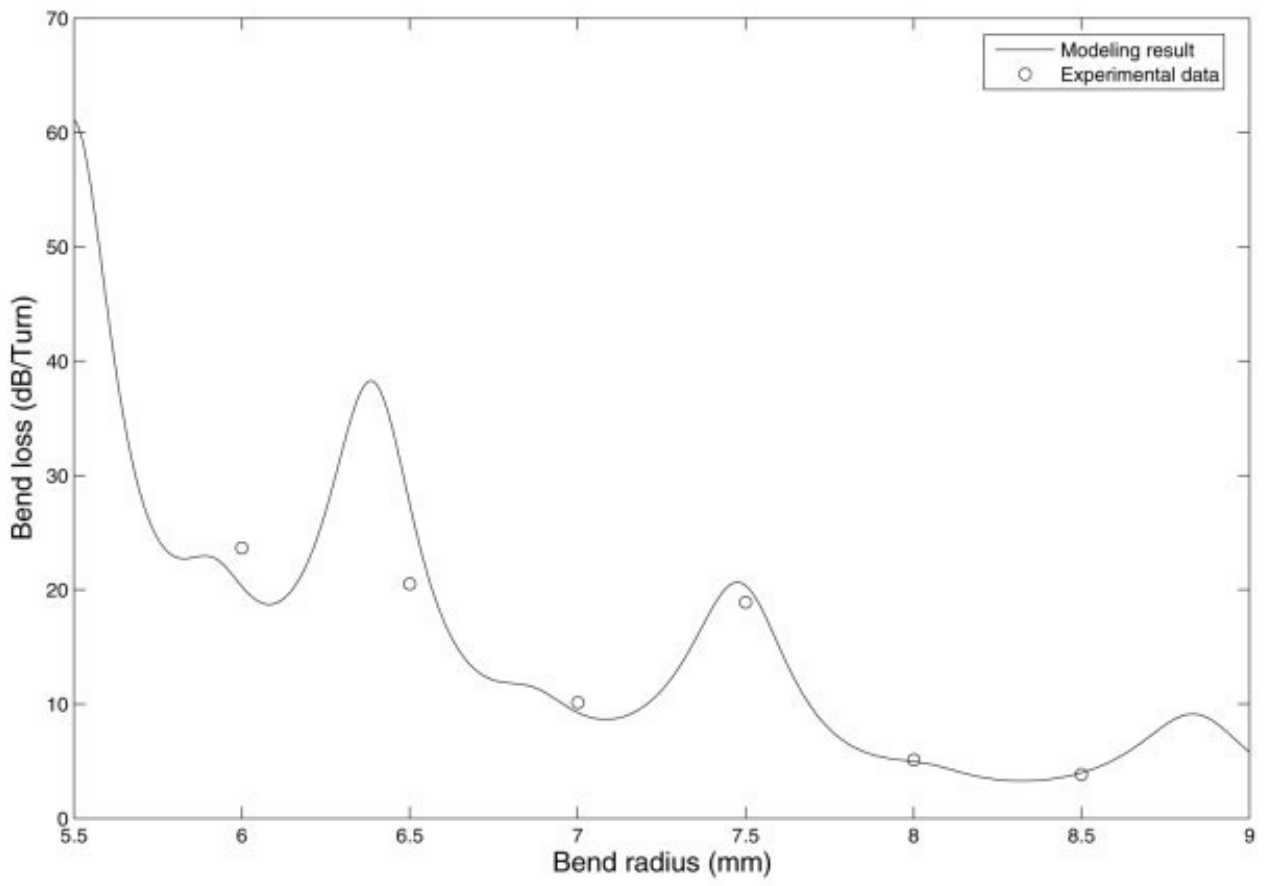

(b)

Figure 3 (a) Modeling and measured macrobending losses for bend radius ranging from 6 to $8.5 \mathrm{~mm}$ at the wavelength of $1500 \mathrm{~nm}$. (b) Modeling and measured macrobending losses for bend radius ranging from 6 to $8.5 \mathrm{~mm}$ at the wavelength of $1600 \mathrm{~nm}$ 


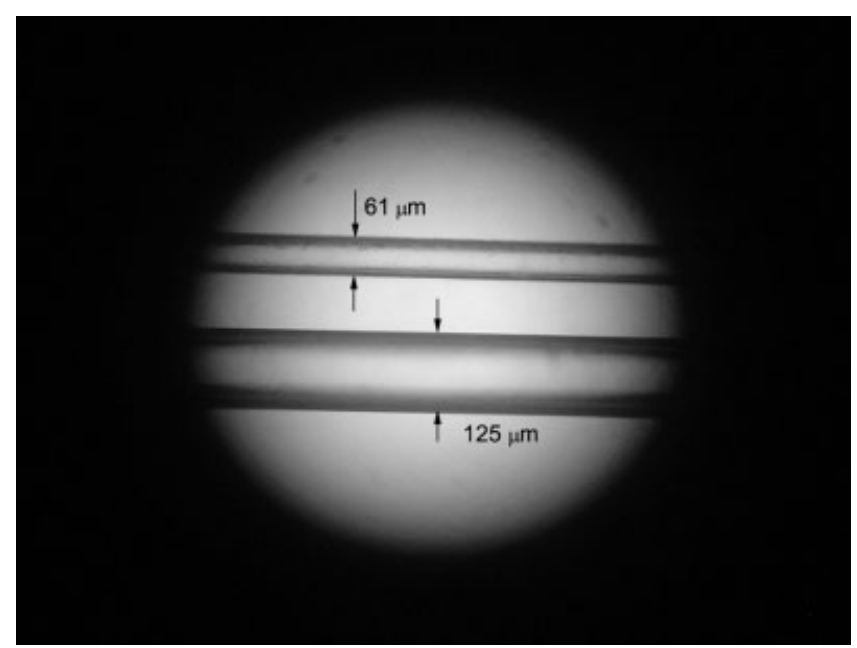

Figure 4 Photograph of etched thinned-cladding fiber contrasting against standard stripped bare SMF28 optical fiber

with the modeling result (solid line) from Ref. [7] and this yields a suitable value of $h$ in the formula (1).

\section{FIBER BEND LOSS WITH COATING LAYERS}

As mentioned in the introduction, the existence of the coating layer(s) will produce a so-called WGM for a bending fiber because of the reflection of the radiated field at the interface between the cladding layer and the coating layer. Previous published investigations of fiber macrobending loss have developed a series of formulas for the calculation of fiber macrobending loss, and when the effect of the coating layer is included, the formulas presented in Ref. [10-12] are used. In this experiment, the single mode fiber was wrapped on a mandrel consisting of multiple sections, each providing a different usable diameter. The bending fiber was connected to a tunable laser and an optical spectrum analyzer. The bend losses of SMF28 fiber with an absorbing layer (to remove the reflection occurring at the interface between the coating layer and air) were measured for the bending radii ranging from 6 to $8.5 \mathrm{~mm}$ when the wavelength is 1500 and 1600 nm, respectively [see Figs. 3(a) and 3(b)].

One can see that as the bend radius increases, the nonmonotonic decrease of both the experimental and theoretical results of the bend loss confirms the impact of a strong WGM influence caused by the coating layer as mentioned earlier. In Figure 3, the modeling results agree well with the experimental data for a bend radius in the range from 7 to $8.5 \mathrm{~mm}$. When the bend radius gets smaller, the agreement between the theoretical and experimental results decreases. One reason is that in the experiment for such cases, the bend loss is highly sensitive to the bend radius, for example, the discrimination is about $40 \mathrm{~dB}$ between the bend radius 5.5 and $6 \mathrm{~mm}$ in Figure 3(b), however, in practice it is difficult to control the bend radius precisely.

\section{BEND LOSS OF A BARE SMF28 AFTER STRIPPING COATING LAYERS AND PARTIALLY ETCHING THE CLADDING LAYER}

In our experiments, the SMF28 fiber coatings were stripped by hot concentrated sulfuric acid $\left(\mathrm{H}_{2} \mathrm{SO}_{4}\right.$, wt $\left.>95 \%, \sim 200^{\circ} \mathrm{C}\right)$. It is found that the fiber is easily broken even when the bend radius is smaller than $10 \mathrm{~mm}$. To reduce the bending stress and retain the mechanical flexibility of bare fiber, the fiber cladding is etched partly by using hydrofluoric (HF) acid. After the etching process using HF acid and cleaning by acetone and alcohol, the diameter of thinned-cladding fiber was about $61 \mu \mathrm{m}$ measured by a high-precision screw micro- meter. Both the bare SMF28 and the thinned-cladding fiber as seen under a microscope are shown in Figure 4.

We measured the bend loss of the bare SMF28 fiber with a diameter of $61 \mu \mathrm{m}$, for a bend radius of 5.5, 6.0, and $6.5 \mathrm{~mm}$ (the bend length is one turn), in the wavelength range from 1500 to $1600 \mathrm{~nm}$, and the corresponding results are presented in Figure 5. For this case, the reflection of the radiated field at the interface between the cladding layer and air has a significant impact on the bend losses. When the bending etched fiber without an absorbing layer coated at the outside, the radiated light is reflected back at the interface between the cladding layer and air. The reflected light is coupled with the propagating light within the core. As distinct from the first case presented in Section 3, where the radiated field is reflected at the interface between the cladding and coating layer, the reflection occurring in the case of etched fiber is much stronger because of the significant refractive index difference between the cladding and air (the etched fiber can be regarded as a multimode fiber when the cladding is treated as the core and the air is the cladding). Because of the strong reflection and recoupling, which can be seen from the simulation results by, for example, the beam propagation method, there is no single quasi-guided mode propagating within the core as the case of bending fiber with a polymer coating. This is why the measured bend loss presented in Figure 5 seems "unusual," for example, from Figure 5, one can see that the bend loss at $5.5 \mathrm{~mm}$ which increases with wavelength to one at 6.5 $\mathrm{mm}$ which decreases, whilst at $6 \mathrm{~mm}$ the loss peaks at $1540 \mathrm{~nm}$.

It should be noted that modeling behavior of the bare etched fiber using the technique outlined in Section 2 is not possible. This can be explained as follows for the case of core-cladding-infinite coating presented in the earlier section, in which the refractive index of coating layer is higher than that of the cladding there is only a single quasi-guided mode propagating in the bending fiber. However, for the bare etched SMF28, the refractive index of the cladding layer is higher than that of the surrounding air and the whole fiber can be regarded as a multimode fiber. The numerical beam propagation method shows the reflected field by the interface of cladding layer and air is strongly coupled with the guided mode within the fiber core along the direction of propagation. No single quasi-guided mode is observed as the case of core-cladding-coating structure (where the refractive index of coating is much higher than that of the cladding layer). For this case, the existing analytical expressions shown in Section 2 are not suitable for modeling.

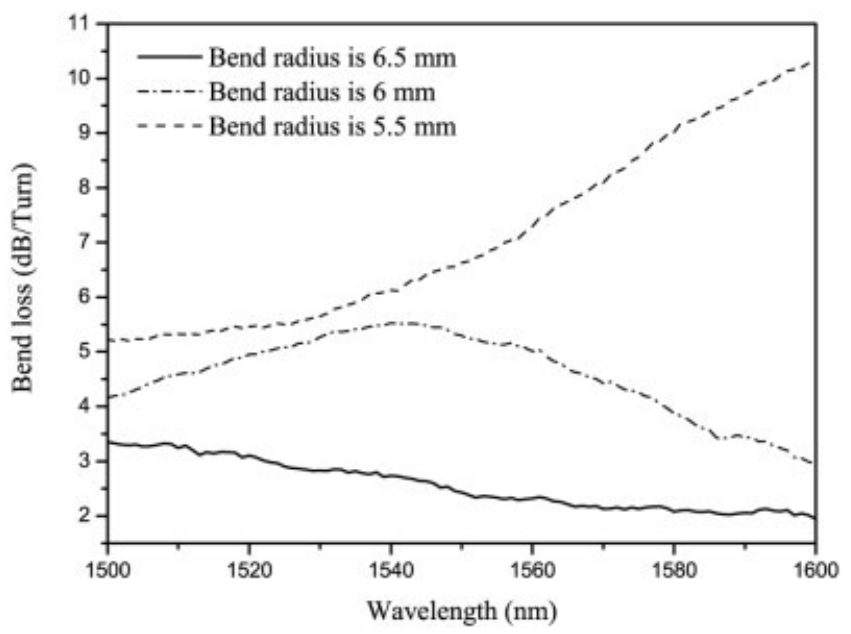

Figure 5 Measured bend loss results of thinned-cladding SMF28 fiber without absorbing layer in wavelength ranging from 1500 to $1600 \mathrm{~nm}$ for bend radius is $5.5,6.0$, and $6.5 \mathrm{~mm}$ 


\section{BEND LOSS FOR THE CORE-CLADDING-ABSORBING LAYER STRUCTURE}

To remove the impact of the reflection at the interface between the cladding and air, the bare thinned-cladding fiber was coated with an absorbing layer. This case is approximately equivalent to a core-infinite cladding structure and the analytical expression for calculating the fiber bend loss with an infinite cladding developed by Marcuse [7, 8] is used.

In the experiment, the etched section coated with absorbing layer was bent to form a small $360^{\circ}$ bend in free space, with the fiber overlapped in a "knot-like" fashion for mechanical stability, and the ends of fiber were connected with a tunable laser and an

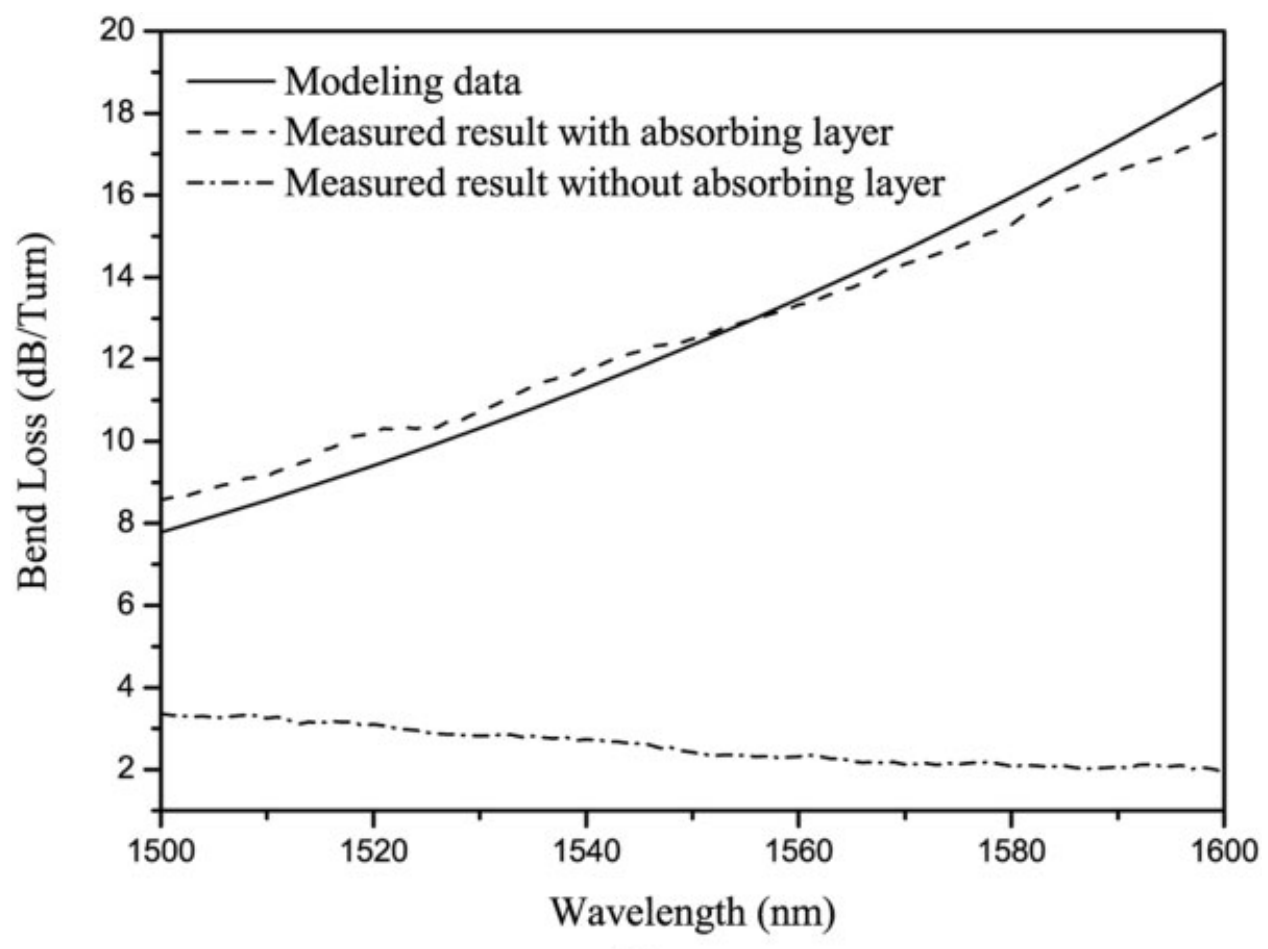

(a)

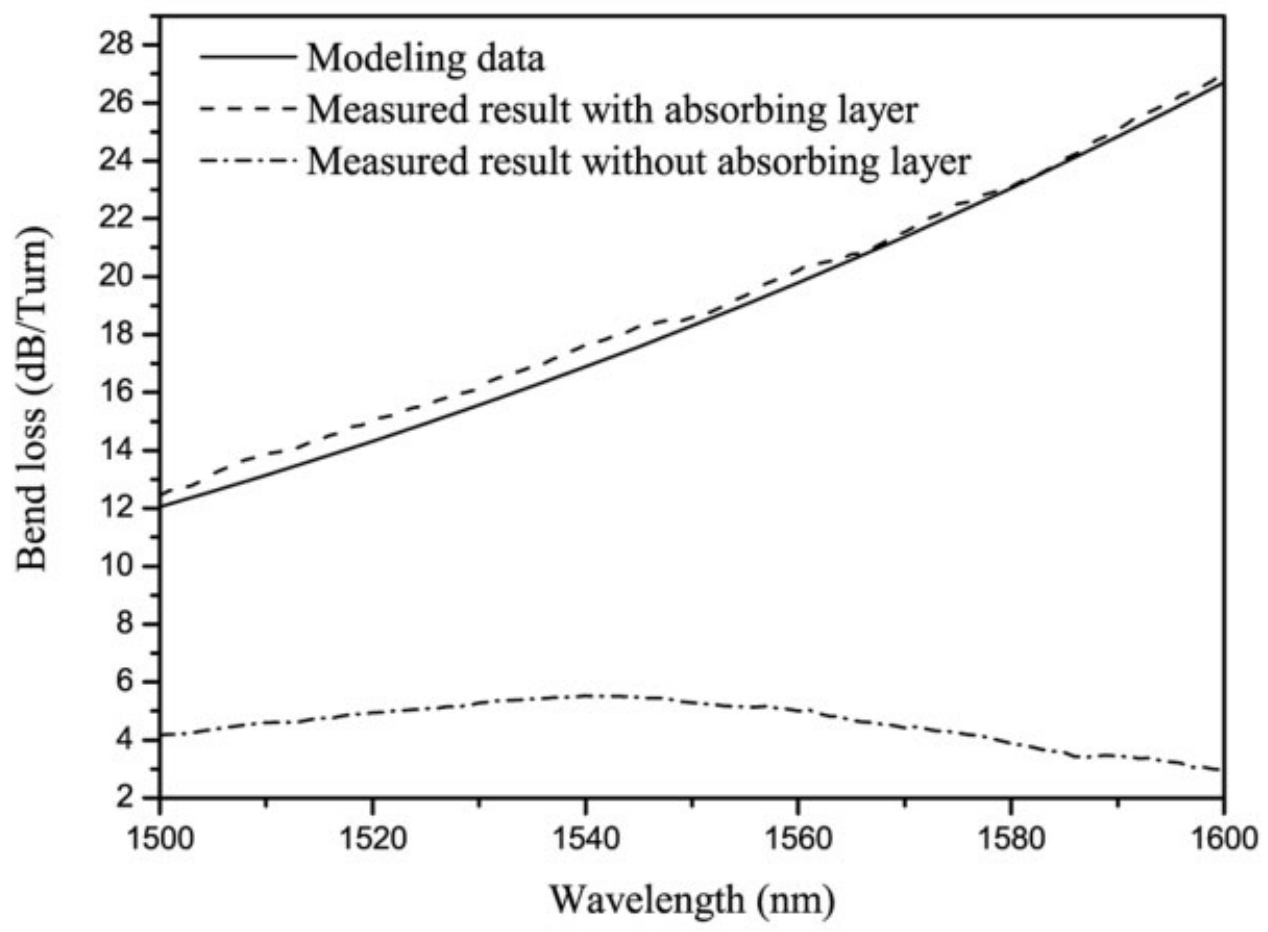

(b)

Figure 6 (a) Measured and modeling bend loss results for thinned-cladding fiber at wavelength range from $1500 \mathrm{~nm}$ to $1600 \mathrm{~nm}$ with bend radius is 6.5 $\mathrm{mm}$ with and without absorbing layer. (b) Measured and modeling bend loss results for thinned-cladding fiber at wavelength range from $1500 \mathrm{~nm}$ to 1600 $\mathrm{nm}$ with bend radius is $6 \mathrm{~mm}$ with and without absorbing layer 
optical spectrum analyzer, respectively. The operating process is the same as the experiment which has been described earlier in Section 4. The theoretical and experimental macrobending loss curves versus wavelength ranging from 1500 to $1600 \mathrm{~nm}$ for bend radius of 6.5 and $6 \mathrm{~mm}$ are presented in Figures 6(a) and 6(b), respectively, from which one can see that the theoretical bend loss agrees with the experimental results. As a comparison, the measured bend losses of bare SMF28 in Figure 5 are also presented. The difference of bend loss between the two cases, i.e., bare SMF28 and the bare SMF28 with an absorbing layer, shows that the reflection occurring at the interface between the cladding layer and air has a significant effect on the bend loss.

\section{CONCLUSION}

In conclusion, we have presented a thorough theoretical and experimental investigation of the macrobending loss characteristics of a standard single mode fiber with small bend radii, which includes theoretical modeling analysis for fiber bend loss, for SMF28 with coating layers and the bare SMF28 after stripping the coating layers and chemical etching of partial cladding. Both experimental and theoretical results have demonstrated the impact of reflection occurring at the interface between the cladding and coating layer or the cladding layer and air on the bend loss.

\section{REFERENCES}

1. N. Lagakos, T. Litovitz, P. Macedo, R. Mohr, and R. Meister, Multimode optical fiber displacement sensor, Appl Opt 20 (1981), 167-176.

2. N. Lagakos, J.H. Cole, and J.A. Bucaro, Microbend fiber-optic sensor, Appl Opt 26 (1987), 2171-2180.

3. M.T. Wlodarczyk, Wavelength referencing in single-mode microbend sensors, Opt Lett 12 (1987), 741-743.

4. R.C. Gauthier and C. Ross, Theoretical and experimental considerations for a single-mode fiber-optic bend-type sensor, Appl Opt 36 (1997), 6264-6273.

5. Y. Jeong, S. Baek, B. Lee, S. Choi, and K. Oh, Macrobend sensor via the use of a hollow-core splice fiber: Theory and experiments, Opt Eng 41 (2002), 1815-1820.

6. S.H. Nam and S. Yin, High-temperature sensing using whispering gallery mode resonance in bent optical fibers, IEEE Photon Technol Lett 17 (2005), 2391-2393.

7. D. Marcuse, Curvature loss formula for optical fibers, J Opt Soc Am 66 (1976), 216-220.

8. D. Marcuse, Bend loss of slab and fiber modes computed with diffraction theory, IEEE J Quantum Electron 29 (1993), 2957-2961.

9. C. Vassallo, Perturbation of an LP mode of an optical fiber by a quasi-degenerate field: A simple formula, Opt Quantum Electron 17 (1985), 201-205.

10. I. Valiente and C. Vassallo, New formalism for bending losses in coated single-mode optical fibers, Electron Lett 25 (1989), 1544 1545 .

11. H. Renner, Bending losses of coated single-mode fibers: A simple approach, J Lightwave Technol 10 (1992), 544-551.

12. L. Faustini and G. Martini, Bend loss in single-mode fibers, J Lightwave Technol 15 (1997), 671-679.

13. Y. Powell-Friend, L. Phillips, T. George, and A. Sharma, A simple technique for investigating whispering gallery modes in optical fibers, Rev Sci Instrum 69 (1998), 2868-2870.

14. Q. Wang, G. Farrell, and T. Freir, Theoretical and experimental investigations of macro-bend losses for standard single mode fibers, Opt Exp 13 (2005), 4476-4484.

15. Q. Wang, G. Farrell, T. Freir, G. Rajan, and P. Wang, Low-cost wavelength measurement based on a macrobending singlemode Fiber, Opt Lett 31 (2006), 1785-1787.

(C) 2007 Wiley Periodicals, Inc.

\section{ON THE DESIGN OF MULTIFREQUENCY DIVIDERS SUITABLE FOR GSM/DCS/PCS/UMTS APPLICATIONS BY USING A PARTICLE SWARM OPTIMIZATION-BASED TECHNIQUE}

\author{
Zaharias D. Zaharis, ${ }^{1}$ Dimitra G. Kampitaki, ${ }^{1}$ \\ Pavlos I. Lazaridis, ${ }^{1,2}$ Anastasia I. Papastergiou, ${ }^{1}$ and \\ Philippe B. Gallion ${ }^{2}$ \\ 1 Department of Electronics, Alexander Technological Educational \\ Institute of Thessaloniki, 57400 Thessaloniki, Greece \\ 2 Département Communications et Electronique, Unité de Recherche \\ Associée au Centre National de la Recherche Scientifique, 820 Ecole \\ Nationale Supérieure des Télécommunications, 46 rue Barrault, \\ 75634 Paris Cedex 13, France
}

Received 19 February 2007

ABSTRACT: A particle swarm optimization-based technique is applied to design dividers that operate in two or more frequency bands at once. The geometry of the dividers is optimized under specific requirements concerning the impedance-matching bandwidth and the complex current distribution on unmatched real or complex terminal loads for each one of the resonant frequencies. The required current distribution on the loads concerns not only the ratio between the current amplitudes but also the phase difference between the currents. Several cases are studied to show the robustness of the particle swarm optimizer as well as the ability of the technique to derive optimal multifrequency structures suitable for GSM/DCS/PCS/UMTS applications. (C) 2007 Wiley Periodicals, Inc. Microwave Opt Technol Lett 49: 2138-2144, 2007; Published online in Wiley InterScience (www.interscience.wiley.com). DOI 10.1002/mop.22658

Key words: multifrequency dividers; multiband dividers; multiband mobile antennas; mobile communications; particle swarm optimization

\section{INTRODUCTION}

Dividers are structures of great interest, especially for feeding networks of wireless or mobile communications systems [1-3]. The two main purposes of a divider are splitting the signal according to the desired split ratio and providing impedance matching inside the frequency range of operation [4]. Of course, a welldesigned divider must satisfy an additional requirement concerning the desired phase difference between the signals applied on the terminal loads. So far, many methods have been proposed for the designing of dividers that satisfy the above requirements [5-29]. Most of these methods consider dividers operating in a single frequency band. However, the need for simultaneous operation in two or more frequency bands led to the designing of dual-frequency $[28,29]$ or multifrequency dividers.

A multifrequency divider must satisfy the earlier-mentioned requirements concerning the desired impedance-matching bandwidth and the desired signal-split ratio, including the phase difference between the signals applied on the terminal loads. The main difficulty in designing such a divider results from the fact that all the above requirements must be satisfied simultaneously in all the frequency bands, considering that the terminal loads are not matched to the main transmission line that feeds the divider. In addition, a multifrequency divider that complies with the above requirements must be easily implemented in practice.

To overcome the above difficulties, the present work introduces a new technique, which makes use of a particle swarm optimization(PSO) based algorithm developed by the authors. The fundamentals of PSO have been discussed in many papers [30-39] and many problems have already been solved by applying PSO-based methods 